This manuscript is a preprint and has not undergone peer-reviewing. Please do not hesitate to contact any of the authors. We welcome feedback. 


\title{
Determination of the spatial distribution of wetting in the pore networks of rocks
}

\author{
Gaetano Garfí ${ }^{1, *}$, Cédric M. John ${ }^{1}$, Maja Rücker ${ }^{2,1}$, Qingyang Lin ${ }^{4,1}$, Catherine Spurin ${ }^{1}$, \\ Steffen Berg ${ }^{5,3,1}$, and Samuel Krevor ${ }^{1}$
}

\author{
${ }^{1}$ Department of Earth Science and Engineering, Imperial College London, London SW7 2AZ, United Kingdom \\ ${ }^{2}$ Department of Mechanical Engineering, Eindhoven University of Technology, PO Box 513, 5600 MB Eindhoven, \\ The Netherlands \\ ${ }^{3}$ Department of Chemical Engineering, Imperial College London, London SW7 2AZ, United Kingdom \\ ${ }^{4}$ State Key Laboratory of Clean Energy Utilization, State Environmental Protection Engineering Center for \\ Coal-Fired Air Pollution Control, Zhejiang University, Hangzhou 310027, China \\ ${ }^{5}$ Shell Global Solutions International B.V., Grasweg 31, 1031HW Amsterdam, The Netherlands \\ *g.garfi17@imperial.ac.uk
}

\begin{abstract}
The macroscopic movement of subsurface fluids involved in $\mathrm{CO}_{2}$ storage, groundwater, and petroleum engineering applications is controlled by interfacial forces in the pores of rocks, micrometer to millimetre in length scale. Recent advances in physics based models of these systems has arisen from approaches simulating flow through a digital representation of the complex pore structure. However, further progress is limited by a lack of approaches to characterising the spatial distribution of the wetting state within the pore structure. In this work, we show how observations of the fluid coverage of mineral surfaces within the pores of rocks can be used as the basis for a quantitative 3D characterisation of heterogeneous wetting states throughout rock pore structures. We demonstrate the approach with water-oil fluid pairs on rocks with distinct lithologies (sandstone and carbonate) and wetting states (hydrophilic, intermediate wetting, or heterogeneously wetting). The resulting 3D maps can be used as a deterministic input to pore scale modelling workflows and applied to all multiphase flow problems in porous media ranging from soil science to fuel cells.
\end{abstract}

\section{Introduction}

Despite the major scientific and societal interest, predictive modelling of subsurface multiphase flow remains out of reach, with a number of canonical problems in $\mathrm{CO}_{2}$ storage, groundwater, and petroleum engineering ${ }^{1-5}$. These problems are characterised by an inability to model observed behaviour, even when uncertainties due to natural variability in rock properties are accounted for or minimised. Central to the difficulty is that the macroscopic fluid movement is controlled by interfacial forces in the pores of rocks, micrometer to millimetre in length scale ${ }^{6,7}$.

Recent progress has thus focused on building models that simulate flow through a digital representation of the complex pore structure ${ }^{8-11}$. These efforts have identified that the pore structure, in combination with the nature of the fluid-fluid and fluid-solid interfacial forces combine to govern the morphology and dynamics of fluid phases as they are conducted through the pore space. This morphology in turn determines the flow properties, the relative permeability and capillary pressure characteristics, necessary for modelling larger scale flow ${ }^{12-17}$. Predicting this morphology, and its evolution in the pore space, is a key next step in developing physics based models of larger scale flow.

An outstanding challenge in this approach is the inscrutability of the wetting state of mineral surfaces throughout the pore structure. The wetting, the relative affinity for one fluid to coat the surface of a solid relative to another, determines the nature of the local capillary force balance controlling the fluid distribution ${ }^{5,18}$. Unlike the pore structure itself, the wetting state cannot be directly observed because it is determined by the combined chemical nature of the mineral surfaces and fluid phases. Observations of the contact angle in situ exhibit significant uncertainty due to pore structure controls, hysteresis, and noise in imaging processing, and cannot be used for spatially resolved wetting determination ${ }^{19-23}$. Pore scale observations can be used to constrain the average wetting state in models based in equilibrium thermodynamics and integral geometry ${ }^{24-26}$. Yet, it is the spatial distribution of wetting states, and not an averaged wetting state, that determines the nature of flow ${ }^{18,27,28}$. The importance of understanding the wetting state and predicting its impact extends beyond consideration of geological materials. It is central to capillary dominated technologies including filters, fuel cells, and fluid wicking and repellant fabrics ${ }^{29,30}$. The absence of spatially resolved wetting characterisation is inhibiting advances in pore scale model predictions of upscaled flow 
a)

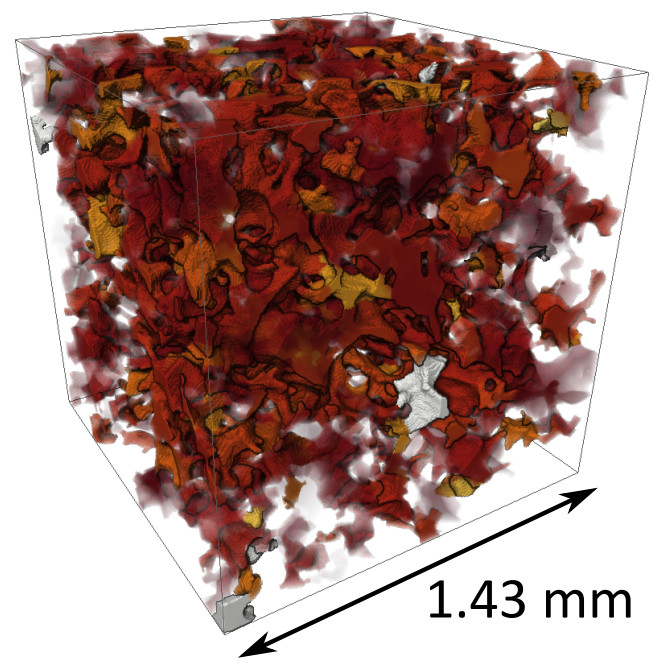

c)

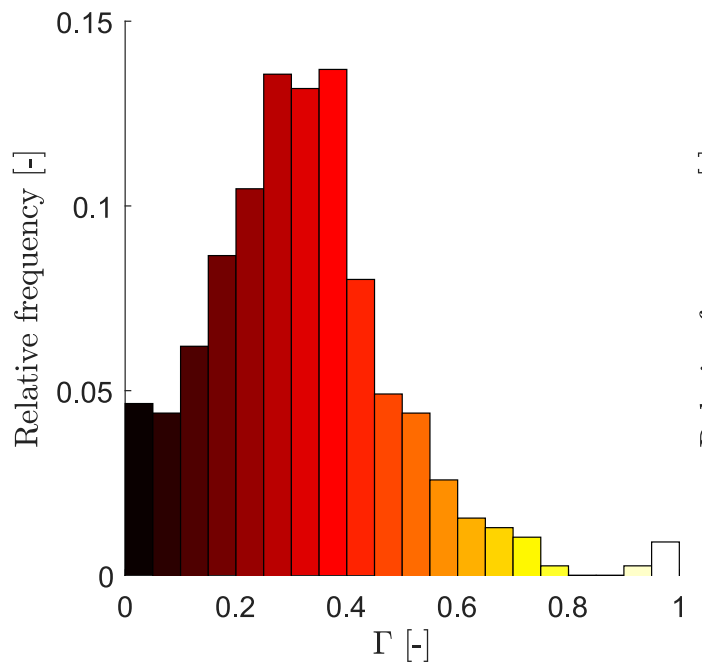

b)

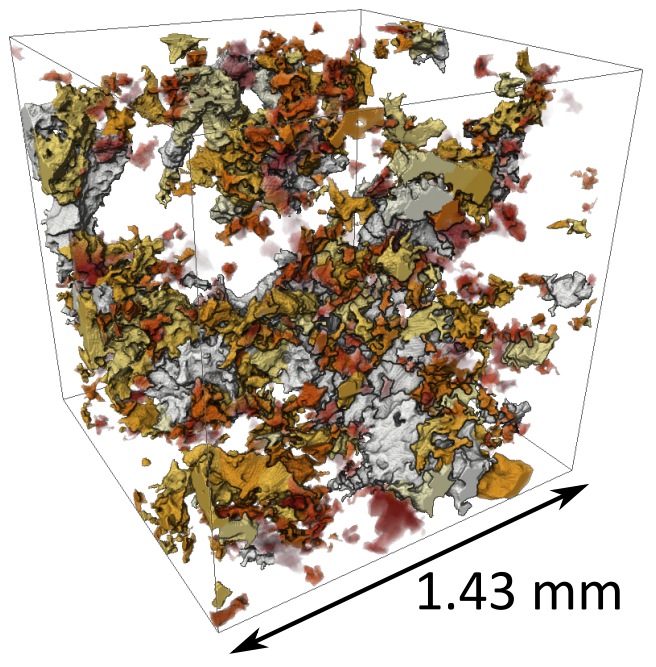

d)

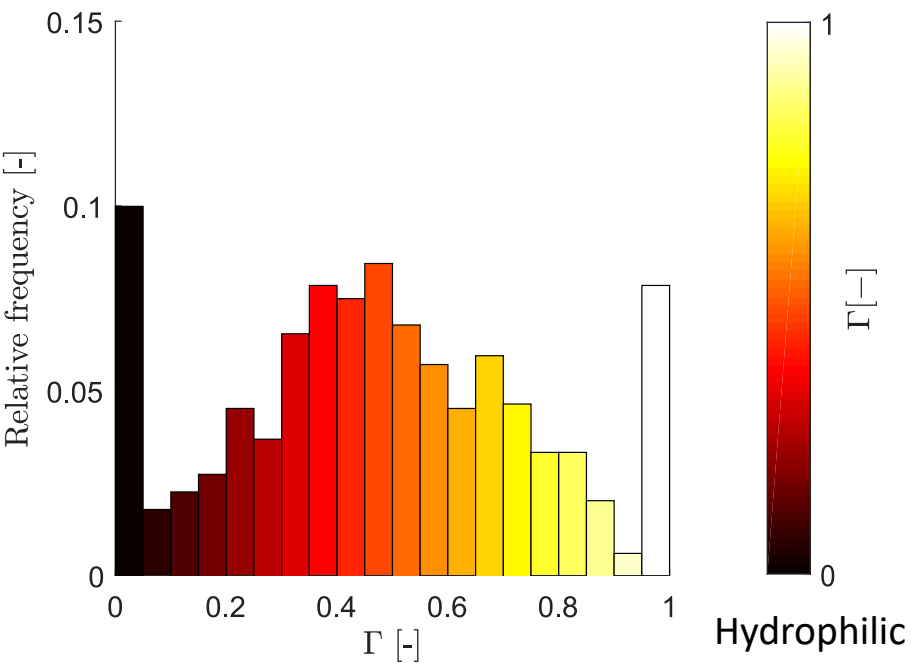

Figure 1. 3D wetting maps of a sandstone (altered Bentheimer sandstone) and a carbonate rock (altered Estaillades limestone). The maps are generated defining and computing a wetting index $(\Gamma)$ on a pore-by-pore basis. The wetting index ranges from 0 to 1 corresponding respectively to two reference wetting cases, hydrophilic, i.e. wetting to the aqueous phase (brine), and hydrophobic, i.e. wetting to the oleic (oil) phase. c) and d) report the relative frequency distributions of the wetting index for altered Bentheimer and altered Estaillades, respectively. The average and standard deviation for altered Bentheimer are $\mu_{\Gamma}=0.316$ and $\sigma_{\Gamma}=0.169$, respectively. The average and standard deviation for altered Estaillades are $\mu_{\Gamma}=0.478$ and $\sigma_{\Gamma}=0.277$, respectively. 
properties, including the use of pore network, Lattice-Boltzmann, and Navier Stokes based flow simulations ${ }^{27,28,31}$.

In this study we overcome these challenges to characterise the wetting state of rocks, spatially resolved at the scale of individual pores (Figure 1). We extend the approach of Garfi et al., $(2020)^{25}$ to show that observations of the relative fluid coverage of rock mineral surfaces with X-ray micro-CT imaging can be used to create a 3D map of the quantified wetting state of the rock. This analysis is performed by measuring fluid phase volumes and fluid-solid interfacial areas in X-ray micro-CT images of rock samples into which two fluid phases have been injected. The workflow is demonstrated for different rock lithologies (sandstone and carbonate rock samples) with different wetting states (uniformly hydrophilic, uniformly intermediate-wetting between the two fluid phases, and heterogeneously wetting). An empirical pore based wetting index is defined based on a thermodynamic model which is calibrated by observations of fluid surface coverage throughout the pore space. This allows us to create 3D wetting maps of the rocks that can be used deterministically in the creation of pore scale numerical models of multiphase flow.

\section{Results}

\section{The macroscopic wetting state and its alteration}

The wetting state of two rock lithologies is investigated in this study: Bentheimer sandstone and Estaillades limestone. For each lithology one dataset comes from a hydrophilic rock sample (most minerals are naturally hydrophilic). A second dataset comes from samples where the wetting state has been altered by a crude oil treatment towards more hydrophobic surfaces within the pore structure.

The four datasets are referred to as either altered or unaltered if treated by crude oil or not. Each dataset consists of one cubic subvolume $\left(1.43^{3} \mathrm{~mm}^{3}\right)$ of one segmented X-ray micro-CT image from cylindrical rock samples that were $6.1 \mathrm{~mm}$ in diameter. The image analysed was selected from among a series of images acquired during the waterflooding or quasi-static co-injection of an oleic phase (an oil) and an aqueous phase (brine) in steps, with the fractional flow of the aqueous phase increasing at each step. The detailed descriptions of the rock lithologies, datasets, flow experiments and imaging are reported in 'Methods'.

The relationship between the oil-coated area fraction $\left(A_{f o}\right)$, i.e. the fraction of mineral surface covered by the oil phase, and the oil volume fraction within a given pore $\left(V_{f o}\right)$ varies by rock lithology and wetting state (Figure 2). The red line in the plots expresses the $A_{f o}=V_{f o}$ relationship (the 1:1 line) and serves as a fixed reference curve for the comparison between datasets. However, note that the distance from this line is only approximately indicative of the wetting state. The precise definition, derived in 'Methods', depends on the pore structure and observations of the fluid distribution. The procedure for measuring these quantities in X-ray micro-CT images is described in 'Methods'.

The unaltered Bentheimer sandstone is homogeneously hydrophilic ${ }^{32}$, and $A_{f o}$ is mostly found below the $1: 1$ line. There is a preferential solid surface coating by brine (Figure 2a)). After the Bentheimer surfaces are altered by crude oil, there is a shift in the data cloud towards the 1:1 line (Figure 2b)). This suggests that a change in wetting preference has occurred and the solid surface wetting has shifted towards a similar preference to both fluid phases. This is frequently referred to as an intermediate wetting state. This finding is consistent with observations reported by others ${ }^{25,32,33}$.

The unaltered Estaillades is also naturally hydrophilic and shows a similar trend as the unaltered Bentheimer, with most data points falling below the 1:1 line (Figure 2c)). A shift towards greater oil-coated area fraction at a given fluid volume fraction is observed for the altered sample (Figure 2d)) than was the case with the Bentheimer. This finding is consistent with past observations of calcite being more prone than quartz to wetting alteration as a result of the interaction with the surface active compounds present in crude oil ${ }^{25,33-35}$. Moreover, there is a wide spread in the area fraction with the data from the altered rock. This suggests a heterogeneous distribution of wetting states within the Estaillades, ranging from pores that remain hydrophilic to pores that obtain a wetting preference to oil after alteration.

The quantitative shifts in both the average values of the data clouds as well as their variation in area fraction are shown by the whisker plots in Figure 2. A bin size of 0.05 in volume fraction units was used. The average standard deviation in the data points is larger for the unaltered Estaillades dataset than for the Bentheimer. This could be a consequence of the additional level of heterogeneity in surface texture and pore geometry Estaillades limestone possesses. The wetting alteration process induces little change in the relative coverage variability observed in the Bentheimer. In contrast, the variability in the altered Estaillades increases dramatically (whisker plots in Figure 2). This reflects the difference in the impact of the wetting alteration process. For the Bentheimer, a uniform but modest change to the wetting state towards an intermediate state is observed. For the Estaillades, a heterogeneously wetting state is obtained with the alteration process.

\section{Pore structure controls on wetting and fluid morphology in individual pores}

The distinct responses of the Bentheimer Sandstone and Estaillades carbonates to wetting alteration appear to be in part a consequence of the pore structure controls on fluid filling. During the alteration process for the Bentheimer the drainage capillary pressure was sufficient for the crude oil to invade most of the pore space uniformly ${ }^{33,36}$. In contrast, the Estaillades 
a)

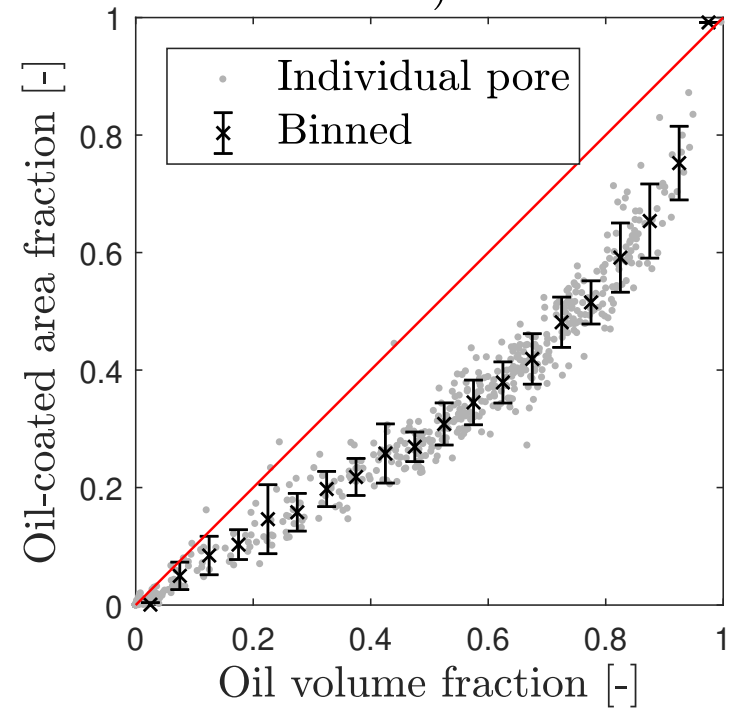

c)

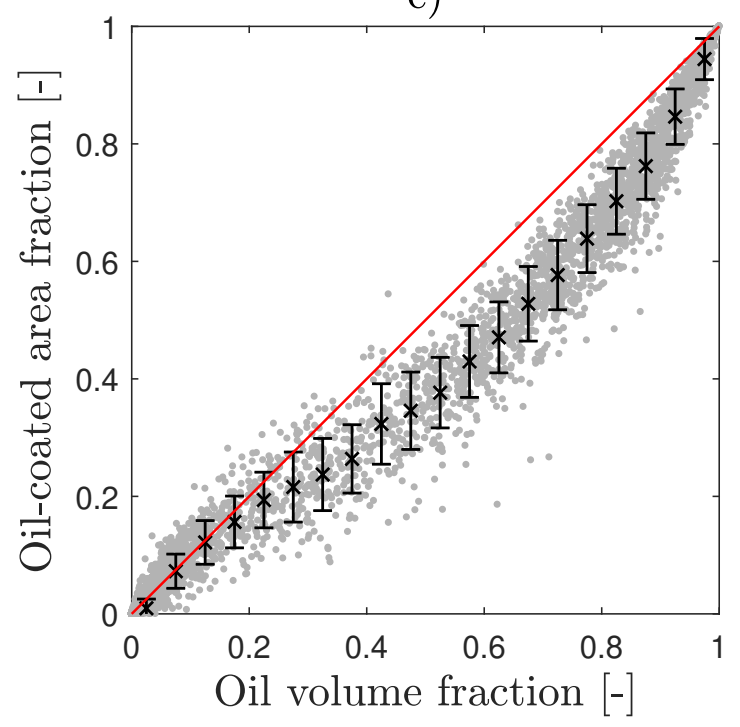

b)

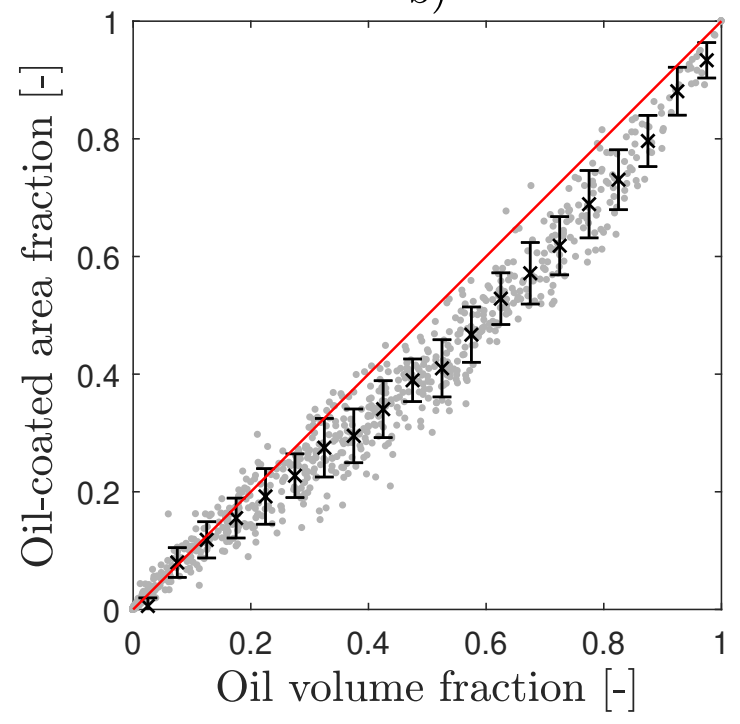

d)

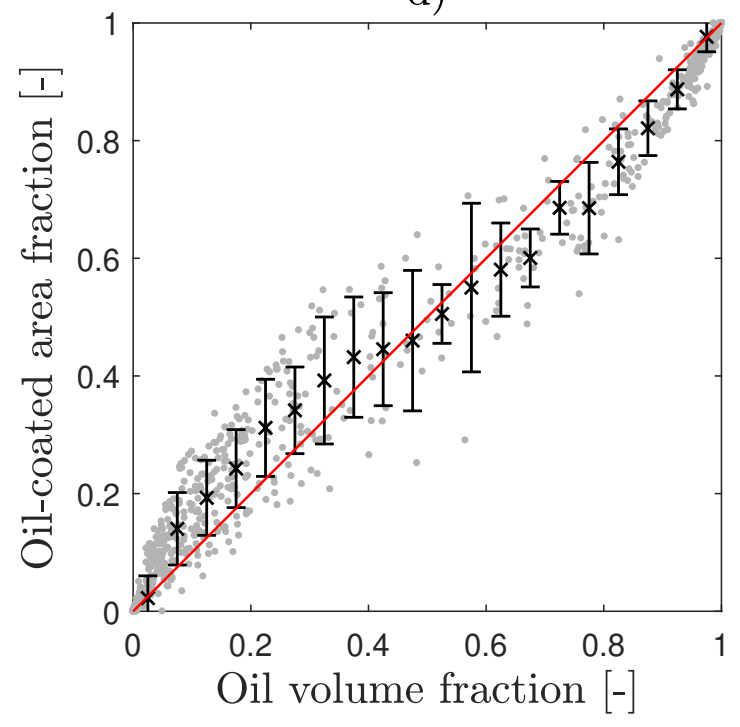

Figure 2. Oil-coated area fraction $\left(A_{f o}\right)$ as a function of oil volume fraction in each identified pore region of the four datasets considered: a) unaltered Bentheimer; b) altered Bentheimer; c) unaltered Estaillades; d) altered Estaillades. The whiskers represent the average and standard deviation computed binning the individual pores on the basis of their oil volume fraction into bins of size 0.05 . The 1:1 line is shown in red. 
has smaller pores and a multi-modal pore size distribution, and only a portion of the pore space was invaded by crude oil during the wetting alteration ${ }^{37,38}$. Figure 3 shows plots of the altered samples with points colored by the size of the pore. The colour represents the ratio between each pore radius and the radius of the largest pore in the region of interest: the brighter the marker, the bigger the pore; the darker, the smaller. The altered Bentheimer does not show any obvious relationship between the size of the pore radii and the fluid surface coverage, i.e., the wetting state. On the other hand, the altered Estaillades shows most of the larger pores (brighter colouring) above the 1:1 line, with a hydrophobic state. The pores become systematically smaller the more hydrophilic they are on the figure. The bigger pores are more likely to be hydrophobic because their surfaces were more readily accessible to the oil phase during the alteration process.

The location of points on the plots identifies pores in which the visualised fluid configurations are characteristically hydrophilic or hydrophobic. Figure 3 shows a selection of the 2D and 3D images of three pores of the altered Estaillades. Their locations on the area fraction plot are shown by black circles in Figure 3. Pore 1 (a large pore, Figure $3 \mathrm{c}$ )-f)-i)) is an example of a hydrophobic pore (from the area fraction graph) with a low oil volume fraction. The oil has been displaced and only left in the narrow corners of the pore space. Pore 2 is another hydrophobic pore (Figure $3 \mathrm{~d}$ )-g)-j)) but is mainly filled with oil, which traps a brine droplet in the centre of the pore space. The shape of the fluid-fluid interface is characteristic of a hydrophobic state. Pore 3 is a smaller pore identified as wetting to water (Figure $3 \mathrm{e}$ )-h)-k)). The fluid configuration exhibits typical hydrophilic behaviour, with the brine snapping-off an oil droplet and securely trapping it at the centre of the pore space. This suggests that the location of a data point on this figure may be used in quantitative characterisation of the wetting state.

\section{Pore by pore wetting maps for the altered rock samples}

We created a dimensionless index, $\Gamma$, assigning a quantitative measure of wetting to individual pores, with the range $0 \leq \Gamma \leq 1$ representing the range from hydrophilicity to hydrophobicity. The interpretation makes use of the oil-coated area fraction and volume fraction data in Figure 2. See 'Methods' for details.

Figure $1 \mathrm{a}$ ) and b) report the relative frequency distributions of the wetting index $\Gamma$ estimates obtained for altered Bentheimer and altered Estaillades. The distribution obtained for the altered Bentheimer is narrow (standard deviation of $\sigma_{\Gamma}=0.169$ ) and positively skewed with an average value of $\mu_{\Gamma}=0.316$. In contrast, the average wetting index for the altered Estaillades is $\mu_{\Gamma}=0.478$ and the distribution is fairly symmetric around the average and heavy tailed $\left(\sigma_{\Gamma}=0.277\right)$. There are also peaks showing a substantial number of pores whose wetting state corresponds or exceeds the reference hydrophilic and hydrophobic models.

These provide a quantitative description of the observations already described qualitatively in the previous section. The altered Bentheimer is at a more homogeneous and intermediate wetting state relative to the altered Estaillades where the pore surface wetting preference ranges from hydrophilic to hydrophobic. The varying degree of heterogeneity in the wetting state is also visually evident in the 3D wetting maps generated from the wetting index (Figure $1 \mathrm{c}$ ) and d)). The maps do not report the individual pore regions for which a wetting index was not estimated, i.e. individual pores completely filled with brine or oil where information about the wetting state cannot be obtained from the single image we considered.

\section{Discussion}

Fluid surface coverage is a wetting state descriptor that can be used at the scale of individual rock pores. In this study the concept is tested with rocks presenting different wetting states, but its application can be extended to other porous media whose wetting state is unknown. An approach is provided whereby a pore based wetting index is created to characterise the wetting state of heterogeneously wet rocks with two rock lithologies and distinct wetting states. The wetting index is based in thermodynamic theory but derived empirically for a given rock-fluid system. It is generated by fitting polynomials to data of surface area coverage and pore volume fraction of fluids in homogeneously hydrophilic datasets. It can subsequently be applied to the interpretation of the wetting state of the same rock type at any other wetting state.

The use of fluid surface coverage overcomes many of the issues limiting previous wetting characterisation approaches using pore scale imagery. This approach relies on the measurement of fewer geometrical features, i.e. fluid-solid interfacial areas and fluid volumes on a single X-ray micro-CT image. Additionally, the features of interest are directly observed, proportional to a number of voxels identified in the segmented imagery, rather than interpreted as with calculations of curvature or contact angle. These features combine to boost the signal and reduce the uncertainty arising from the image processing and interpretation ${ }^{23}$. The increase in signal arising from these simplifications in the observation is what allows for the use of the measure at a high enough spatial resolution to perform the analysis at the scale of tens of micrometres. Moreover, the observation itself only requires imagery of a rock with two fluid phases distributed throughout the pore space. This avoids complications that might arise from requirements to track pore scale events or dynamics during changes in saturation state.

In this study, the wetting index estimates identified differing characteristics of the wetting alteration by crude oil of two rock samples and could be used to identify pores with fluid arrangements characteristic of various wetting states. This has confirmed that the analysis of fluid surface coverage on a pore-by-pore basis allows for the direct identification of the degree of 
a)

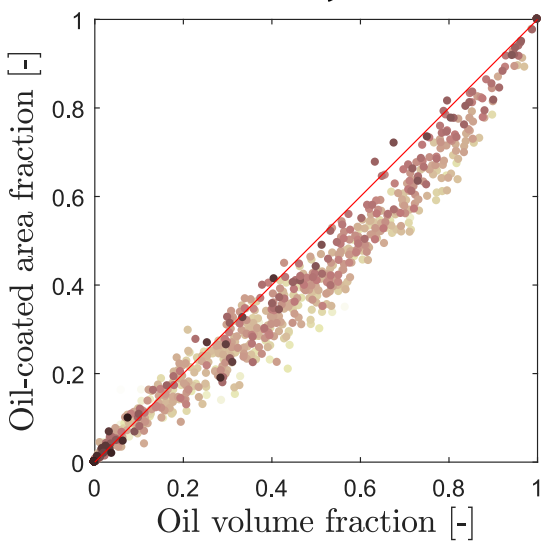

b)

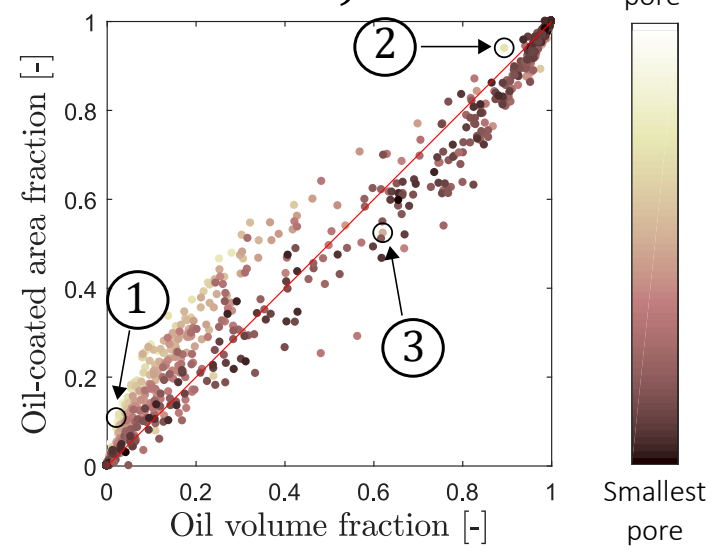

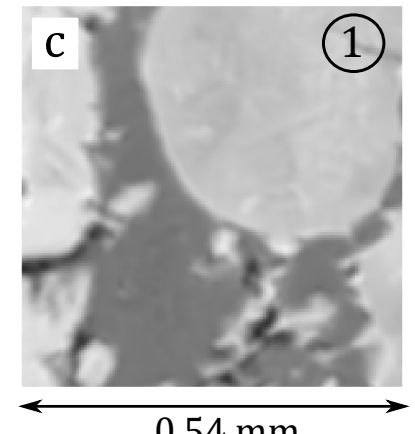

$0.54 \mathrm{~mm}$
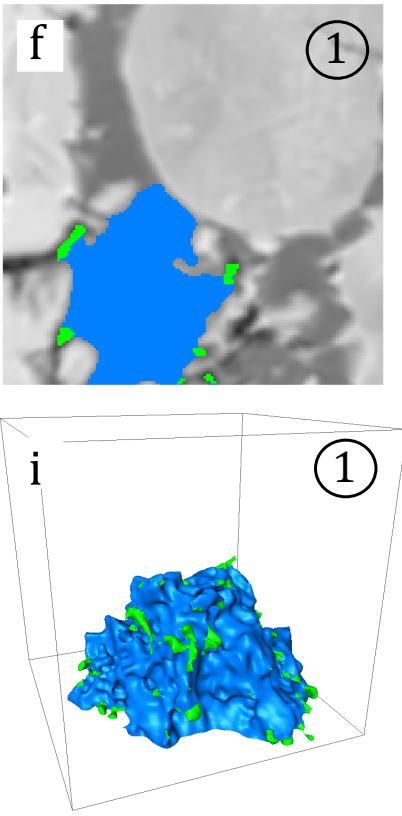
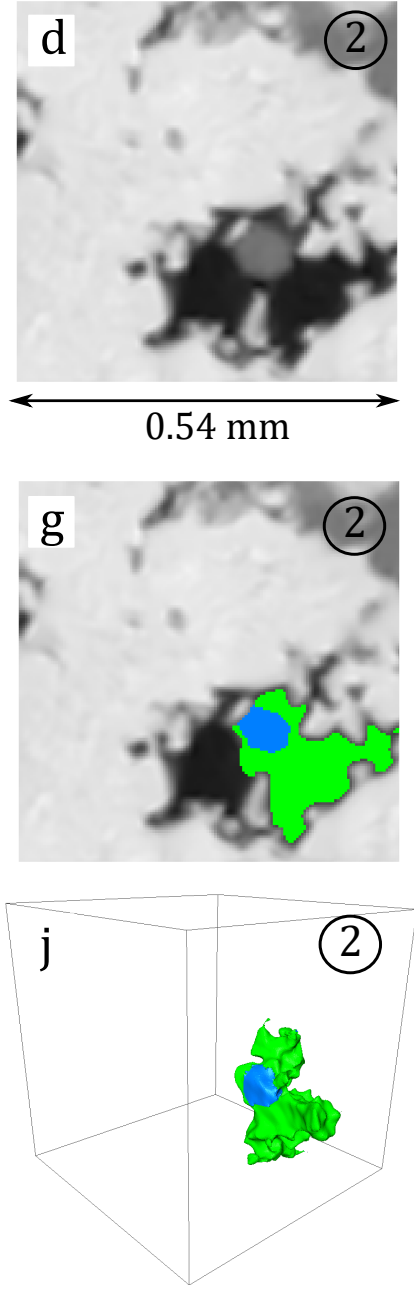

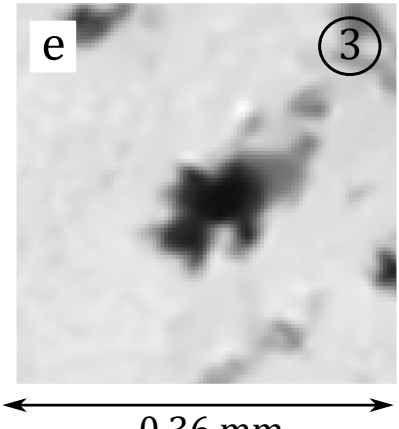

$0.36 \mathrm{~mm}$
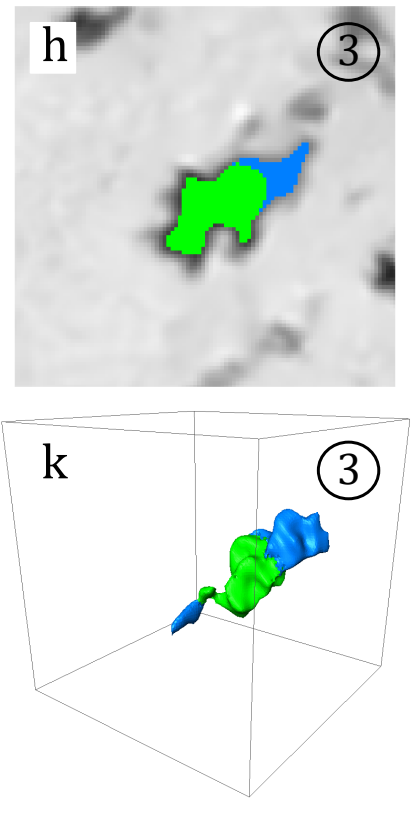

Figure 3. Relationship between fluid surface coverage and pore size (a) altered Bentheimer, b) altered Estaillades) and selected fluid arrangement in three pores (1-2-3) of altered Estaillades (c) to k)). The oil-coated area fraction $\left(A_{f o}\right)$ is reported against the oil volume fraction of each pore. The marker colour represents the relative size of the pore to the largest pore in the region of interest. The darker, the smaller the pore. The relative surface coverage of pores 1,2 and 3 are highlighted in b) by black circles. Columns from left to right: a hydrophobic pore (1) where brine displaced most of the oil (c), f), i)); another hydrophobic pore (2) where oil trapped a brine droplet (d), g), j)); a hydrophilic pore (3) in which brine snapped-off an oil droplet (e), h), k)). 
heterogeneity in the wetting state. This advances our ability to characterise wetting in the way required for further advancement in pore scale modelling approaches. The pore based wetting index allows for the creation of $3 \mathrm{D}$ wetting maps. This is a long sought goal in pore scale modelling. Where previously the input of wetting state distributions has been used as a tuning parameter, the distribution can now be input deterministically, giving rise to the potential for predictive pore scale modelling approaches $^{27,31,39}$.

\section{Methods}

\section{Rock lithologies and datasets}

Bentheimer sandstone is a well sorted sandstone comprising $98 \mathrm{wt} \%$ quartz and a total 2 wt $\%$ of kaolinite, chlorite and microcline. Estaillades limestone is a carbonate rock with a typical calcite content of $98 \mathrm{wt} \%$ and 2 wt $\%$ quartz. For each lithology two datasets are analysed. For both lithologies two rock samples are considered, one in its natural wetting state, and one involving the treatment of the rock sample with crude oil (Table 1). This process is known as ageing and is designed to reproduce wetting alteration processes observed in hydrocarbon deposits ${ }^{40-42}$. Each dataset consists of X-ray micro-CT images coming from experiments performed on a single rock sample. The datasets related to treated rock samples will be referred to as altered, while the untreated ones as unaltered.

\section{Bentheimer Sandstone: unaltered and altered}

The Bentheimer sandstone datasets were previously created from laboratory observations and described in ${ }^{32}$ and ${ }^{33}$. Both datasets consists of the X-ray micro-CT images of quasi-static fractional flow co-injection experiments performed in cylindrical rock samples with a diameter of $6.1 \mathrm{~mm}$ (brine fractional flow, $f_{b}=Q_{b} /\left(Q_{o}+Q_{b}\right)$, with $Q_{i}$ being the flow rate of the fluid phase- $i$ ). The oil-phase (decalin) drainage was performed into the brine saturated sample by centrifugation with a rotation speed such that the capillary pressure obtained was sufficient to reach irreducible water saturation (rotation speed of 3200 $\mathrm{rpm}$, corresponding to a capillary pressure of $60 \mathrm{kPa})^{36}$. In the unaltered Bentheimer, the imbibition was performed right after drainage in capillary dominated conditions. Brine imbibition was conducted in seven steps, increasing the fractional flow of brine from 0 to $1\left(f_{b}=\{0,0.05,0.15,0.30,0.50,0.85,1\}\right)$. The micro-CT images were acquired at the end of each fractional flow step once the pressure gradient across the sample equilibrated. For each dataset our analysis focuses on cubic regions of interest of $400^{3}$ voxels, with a voxel size of $3.58 \mu \mathrm{m}$ corresponding to $1.43^{3} \mathrm{~mm}^{3}$ of the image acquired at fractional flow $f_{b}=0.50$.

The ageing procedure employed for the altered Bentheimer dataset was performed between drainage and the co-injection fractional flow steps of oil and brine. After the drainage process, the sample was submerged in crude oil resulting in a replacement of decalin by crude oil through diffusion. The sample was then stored immersed in crude oil for 30 days at $80^{\circ} \mathrm{C}$. After this alteration process, the sample was submerged in decalin to replace the crude oil. Subsequently the coreflood experiment was performed. The micro-CT images were acquired after eight fractional flows $\left(f_{b}=\{0,0.02,0.06,0.24,0.50,0.80,0.90,1\}\right)$. The brine composition was $3.5 \mathrm{wt} \%$ for the untreated sample and $3.5 \mathrm{wt} \% \mathrm{KI}, 1.09 \mathrm{wt} \% \mathrm{NaCl}, 0.02 \mathrm{wt} \% \mathrm{MgCl}_{2} .6 \mathrm{H}_{2} \mathrm{O}, 0.11 \mathrm{wt} \%$ $\mathrm{CaCl}_{2} \cdot 2 \mathrm{H}_{2} \mathrm{O}$ for the treated one.

\section{Unaltered Estaillades Limestone}

An Estaillades core of $6.1 \mathrm{~mm}$ diameter and $25 \mathrm{~mm}$ length was chosen. The sample was first saturated under vacuum with brine (12.68 wt $\left.\% \mathrm{NaCl}, 5.32 \mathrm{wt} \% \mathrm{CaCl}_{2} \cdot 2 \mathrm{H}_{2} \mathrm{O}, 2.72 \mathrm{wt} \% \mathrm{MgCl}_{2} \cdot 6 \mathrm{H}_{2} \mathrm{O}\right)$. Consequently, decalin drainage was obtained by centrifugation, as was done previously for the Bentheimer samples ${ }^{36}$. However, in this case the rotation speed was chosen so that the brine saturation at the end of drainage was larger than the irreducible one (rotation speed of $1700 \mathrm{rpm}$, corresponding to a capillary pressure of $26 \mathrm{kPa}$ ). The decalin was then displaced by diffusion with decane, which was the oil phase used throughout the flow experiment. The waterflooding experiment was performed in a flow loop similar to that described in ${ }^{32}$, with the difference that no differential pressure was measured across the sample and the brine and oil pumps were used for separate injection steps. A confining pressure of $5 \mathrm{MPa}$ was applied and the receiving pump pressure was set to $3 \mathrm{MPa}$. First, decane was injected at a constant flow rate of $0.015 \mathrm{ml} / \mathrm{min}$, to obtain a connected fluid phase throughout the entire flow loop. The flow rate was low enough that no further brine would be displaced. Then, around 40 pore volumes of brine were injected into the rock sample at a flow rate of $0.015 \mathrm{ml} / \mathrm{min}$ (capillary dominated conditions).

We used an FEI Heliscan microCT to image the entire cross section of the sample over a length of $6 \mathrm{~mm}$, with a voxel size of $2.39 \mathrm{~mm}$. The X-ray source voltage was set to $95 \mathrm{kV}$ and the tube current to $70 \mathrm{~mA}$. The raw projections were reconstructed employing an iterative backprojection algorithm provided by the scanner's manufacturer. The first image acquisition was performed after drainage, with the brine and the decane having no obvious contrast in the greyscale intensity. For the waterflooding step, part of the $\mathrm{NaCl}$ was substituted with $\mathrm{NaI}\left(7 \mathrm{wt} \% \mathrm{NaI}, 5.68 \mathrm{wt} \% \mathrm{NaCl}, 5.32 \mathrm{wt} \% \mathrm{CaCl}_{2} .2 \mathrm{H}_{2} \mathrm{O}, 2.72 \mathrm{wt} \%\right.$ $\mathrm{MgCl}_{2} \cdot 6 \mathrm{H}_{2} \mathrm{O}$ ) in order to obtain contrast between the brine and the decane phases.

The drainage image was segmented by watershed segmentation ${ }^{43}$ into two phases: rock and pore space. The Estaillades is known to usually present around $50 \%$ of the porosity at length scales below the typical resolution range of lab-based micro-CT 
Table 1. Physical and chemical properties of the crude oils employed in the wetting alteration protocol. The listed properties are: density $(\rho)$, viscosity $(\mu)$, total acid number (TAN), total basic nitrogen (TBN). The results of the SARA analysis (saturates, aromatics, resins and asphaltenes) are reported as well.

\begin{tabular}{ccc}
\hline & Treated rock lithology & \\
\hline & Bentheimer & Estaillades \\
$\rho\left[\mathrm{kg} / \mathrm{m}^{3}\right]$ & 859 & 834 \\
$\mu[\mathrm{mPas}]$ & 9.4672 & 4.8731 \\
TAN $[\mathrm{mgKOH} / \mathrm{g}]$ & 0.09 & 0.07 \\
TBN $[\mathrm{mg} / \mathrm{kg}]$ & 270.6 & 83.9 \\
Sat $[\mathrm{wt} . \%]$ & 44 & 58.45 \\
Aro [wt. $\%]$ & 44 & 36.92 \\
Res [wt. $\%]$ & 9.69 & 4.36 \\
Asp [wt.\%] & 2.31 & 0.28 \\
\hline
\end{tabular}

scanners. Although in literature it is possible to find ways to tackle this imaging limitation ${ }^{44}$, in this study we will neglect this contribution to the sample pore space, because the pore-by-pore fluid coverage analysis we propose would still not be possible. The binary segmented drainage image was then used to mask out the rock matrix in the waterflooding image, leading to the segmentation of the brine and decane phases by simple thresholding. In the results section, we performed the analysis of a region of interest of $1.43^{3} \mathrm{~mm}^{3}$, corresponding to $600^{3}$ voxels (voxel size $2.38 \mu \mathrm{m}$ ).

\section{Altered Estaillades Limestone}

The experimental details and original description of the original dataset can be found $\mathrm{in}^{38,45}$. The fluids and the drainage process used for the altered Estaillades were the same as for the unaltered Estaillades. The altered and unaltered Estaillades $6.1 \mathrm{~mm}$ diameter rock cores were sampled from the very same larger core, and the drainage process was performed simultaneously, as described $\mathrm{in}^{36}$. However, in this case, a wetting alteration protocol by means of crude oil exposure followed the drainage process. The decalin was replaced by crude oil through diffusion and the sample was stored immersed in crude oil in a vessel at $3 \mathrm{Mpa}$ and placed into an oven at $80^{\circ} \mathrm{C}$ for 28 days. After this wetting alteration procedure, a quasi-static fractional flow co-injection experiment, analogous to those performed for the two Bentheimer samples, was conducted. The crude oil in the sample was displaced firstly by decalin thorugh diffusion and subsequently decane displaced decalin again through diffusion. The sample was mounted in the same coreflood cell and the same flow loop and imaging facilities used in ${ }^{32,33}$ were employed. The experiment consisted of 7 fractional flow steps $\left(f_{b}=\{0,0.05,0.25,0.50,0.75,0.90,1\}\right)$ with a total flow rate of $0.02 \mathrm{ml} / \mathrm{min}$. The reconstructed tomograms were processed as for the Estaillades Unaltered. Analogous to the other datasets, the region of interest considered in this study was $400^{3}$ voxels, corresponding to $1.43^{3} \mathrm{~mm}^{3}$ of the X-ray micro-CT image acquired at fractional flow $f_{b}=0.50$.

\section{Pore-by-pore fluid surface coverage with X-ray micro-CT}

$\mathrm{X}$-ray micro-CT imaging can be used to visualise the details of multiphase fluid arrangement within the pore space of rock samples $^{46,47}$. After the acquisition and reconstruction of the greyscale 3D images, the final result of the image processing pipeline is a segmented 3D image. The segmentation process consists of assigning each voxel (3D pixel) of the image to an individual phase identified by a numerical index, which commonly represents a certain fluid or solid of interest. In this work, all the segmented X-ray micro-CT images consists of three phases: the brine phase, the oil phase and the rock phase.

The first step of the pore-by-pore wetting state characterisation workflow consists of the identification and separation of the individual pore regions within the X-ray image of the rock. This separation is obtained by a watershed segmentation of the distance map of the pore space. The pore space image is thus divided into $N$ pores, where the voxels constituting each pore- $n$ are assigned the value $n$. The network extraction algorithm we employed also associates a sphere (with radius equal to the maximum ball inscribable into the pore region) and two throats to each pore region ${ }^{48,49}$. By masking the oil-brine-rock image with the image of the separated pores, it is possible to obtain the fluid configuration in each pore region. For each pore region, the fluid-fluid and fluid-solid interfaces are identified and extracted by a voxelized surface generation algorithm. These surfaces are smoothed with an iterative (150 iterations) algorithm preventing boundary shrinking ${ }^{50}$. The smoothed surfaces are measured to define fluid-solid interfacial areas per unit of pore volume $\left(A_{i}\right)$. Finally, by dividing the last by the total interfacial area per unit of pore volume exposed by a pore, one can define the fractions of solid surface coated by oil or brine $\left(A_{f i}\right)$. 


\section{The thermodynamic basis for the fluid-solid interfacial area as a measure of wetting}

The basis for the interpretation of the observations is the thermodynamic model of ${ }^{25}$ describing the specific interfacial area (surface area per unit volume of pore space) between a non-wetting phase and the solid surface $\left(A_{n w s}\right)$ of a porous media as a function of the non-wetting phase saturation $\left(S_{n w}\right)$

$$
A_{n w s}\left(S_{n w}\right)=\frac{\sigma_{n w w}}{\sigma_{n w s}-\sigma_{w s}}\left(2 \int_{S^{\prime}=0}^{S_{n w}} \kappa\left(S_{n w}\right) \mathrm{d} S^{\prime}-A_{n w w}\right),
$$

where $A_{i j}$ is the specific surface area of the interface between phase $i$ and phase $j, S_{i}$ is fluid- $i$ saturation, $\sigma_{i j}$ is the interfacial tension between phase $i$ and phase $j$ and $\kappa$ is the mean fluid-fluid interfacial curvature.

By following the same approach described in ${ }^{25}$ it is possible to derive an analogous relationship expressed in terms of the wetting phase. Let us consider a porous medium with two fluid phases, one wetting $(w)$ and one non-wetting ( $n w)$. With the solid surface, $s$, per unit volume of pore space, the reversible work required to increase the saturation of the wetting phase $w$ can be determined by an energy balance ${ }^{51,52}$ :

$$
\left(p_{w}-p_{n w}\right) \mathrm{d} S_{w}=-p_{c} \mathrm{~d} S_{w}=\sigma_{n w w} \mathrm{~d} A_{n w w}+\sigma_{n w s} \mathrm{~d} A_{n w s}+\sigma_{w s} \mathrm{~d} A_{w s}
$$

where $p_{i}$ is the pressure of the $i$-fluid phase, and $p_{c}$ is the capillary pressure across the fluid-fluid interface. By rearranging the terms and substituting $-\mathrm{d} A_{n w s}=\mathrm{d} A_{w s}$

$$
\frac{\mathrm{d} A_{w s}}{\mathrm{~d} S_{w}}=\frac{1}{\sigma_{n w s}-\sigma_{n w s}}\left(p_{c}+\sigma_{n w w} \frac{\mathrm{d} A_{n w w}}{\mathrm{~d} S_{w}}\right)
$$

Integrating across the same saturation change of Eq. 1 and recalling Young-Laplace equation $p_{c}=2 \kappa \sigma_{n w w}$

$$
A_{w s}\left(S_{w}\right)=\frac{\sigma_{n w w}}{\sigma_{n w s}-\sigma_{w s}}\left(2 \int_{S^{\prime}=1}^{1-S_{w}} \kappa\left(S_{w}\right) \mathrm{d} S^{\prime}+A_{n w w}\left(S_{w}\right)\right)
$$

We can take Eq. 1 and Eq. 4 further by assuming functional forms for $A_{n w w}\left(S_{i}\right)$ and $\kappa\left(S_{i}\right)$. Note that we do this for illustrative purposes, and the wetting characterisation is not dependent on any particular form. Let $A_{n w w}\left(S_{i}\right)$ be a downward parabola with zero specific interfacial area for $S_{i}=0$ and $S_{i}=1^{53,54}$ and maximum in $S_{i}=0.5$ :

$$
A_{n w w}\left(S_{i}\right)=A_{\max }-4 A_{\max }\left(S_{n w}-0.5\right)^{2}
$$

In the case of the non-wetting phase model, a power-law like model is assumed to describe $\kappa\left(S_{n w}\right)$ :

$$
\kappa\left(S_{n w}\right)=\kappa_{e}+\alpha S_{n w}^{n}
$$

and for the wetting-phase, for simplicity, the same model with parameters of opposite sign

$$
\kappa\left(S_{w}\right)=-\kappa_{e}-\alpha S_{w}^{n}
$$

where $\kappa_{e}$ is the mean curvature value in correspondence of the entry capillary pressure (for the wetting phase that value is negative), and $\alpha$ controls the mean curvature value when $S_{n w}$ approaches 1 . Substituting the mean curvature models into Eq. 1 and Eq. 4 and solving the integrals leads to

$$
\begin{aligned}
& A_{n w}\left(S_{n w}\right)=\frac{\sigma_{n w w}}{\sigma_{n w s}-\sigma_{w s}}\left(2 k_{e} S_{n w}+\frac{\alpha}{n+1} S_{n w}^{n+1}-A_{n w w}\left(S_{n w}\right)\right) \\
& A_{w}\left(S_{w}\right)=\frac{\sigma_{n w w}}{\sigma_{n w s}-\sigma_{w s}}\left(2 k_{e} S_{w}-\frac{\alpha}{n+1}\left(\left(1-S_{w}\right)^{n+1}-1\right)+A_{n w w}\left(S_{w}\right)\right)
\end{aligned}
$$

With special reference to applications where the extent of the interfacial areas are directly measured by an imaging technique, a correction factor has to be introduced to take into account the imaging resolution limit. In fact, while the fluid-fluid interfaces are expected to be smooth, the fluid-solid ones are in general rough and various complex fluid arrangements may be observed ${ }^{37}$. As a consequence, the equations can be rewritten as:

$$
A_{n w}\left(S_{n w}\right)=\frac{1}{\beta} \frac{\sigma_{n w w}}{\sigma_{n w s}-\sigma_{w s}}\left(2 k_{e} S_{n w}+\frac{\alpha}{n+1} S_{n w}^{n+1}-A_{n w w}\left(S_{n w}\right)\right)
$$




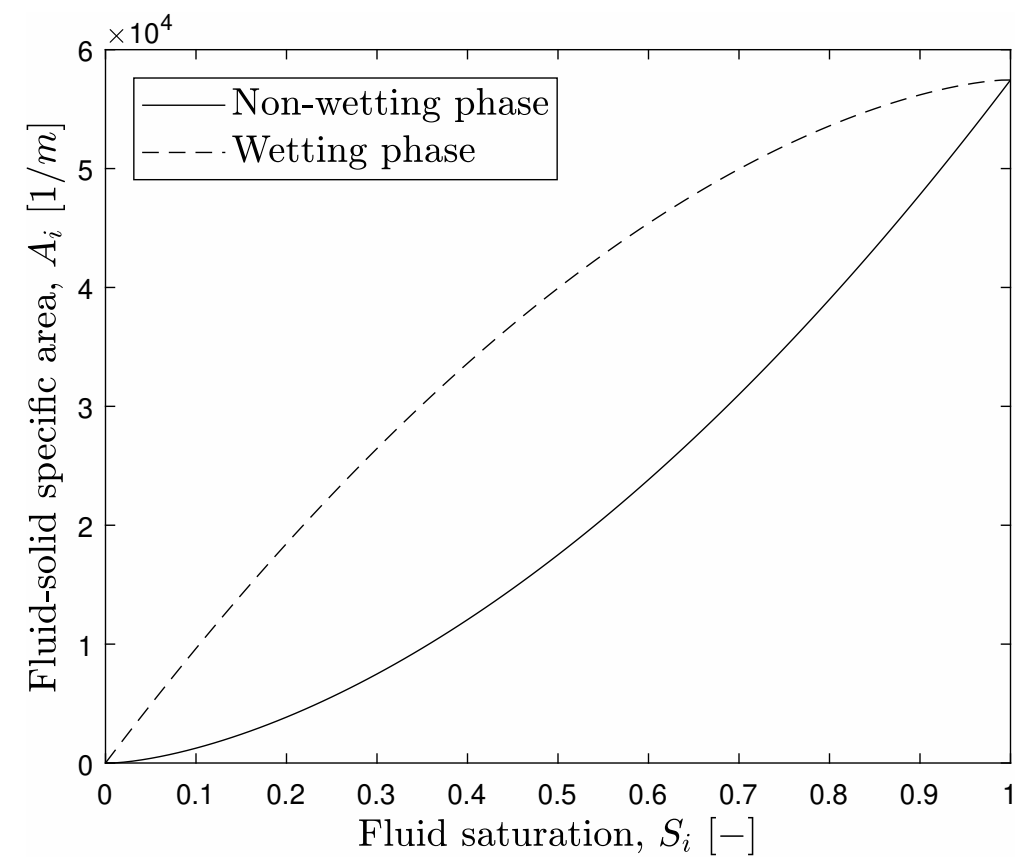

Figure 4. Wetting phase-solid and non-wetting phase-solid specific interfacial areas. For any given value of the saturation, the wetting phase-solid specific interfacial area is larger. The input parameters are: $\kappa_{e}=150001 / \mathrm{m}, \alpha=400001 / \mathrm{m}, n=0.15$, $A_{\max }=160001 / \mathrm{m}$ and $\frac{\sigma_{n w w}}{\sigma_{n w s}-\sigma_{w s}}=1.16$ (i.e. intrinsic contact angle of $30^{\circ}$ ).

$$
A_{w}\left(S_{w}\right)=\frac{1}{\beta} \frac{\sigma_{n w w}}{\sigma_{n w s}-\sigma_{w s}}\left(2 k_{e} S_{w}-\frac{\alpha}{n+1}\left(\left(1-S_{w}\right)^{n+1}-1\right)+A_{n w w}\left(S_{w}\right)\right)
$$

where $\beta=\frac{A_{i, \text { Real }}}{A_{i}}$ and $\beta \geq 1$. Figure 5 reports the theoretical behaviour of the fluid-solid specific interfacial area model for example input values of the model parameters and interfacial tensions ratio $\frac{\sigma_{n w w}}{\sigma_{n w s}-\sigma_{w s}}=1.16$, correspondent to an intrinsic contact angle of $30^{\circ}$ in a smooth capillary tube. Given a certain $S_{i}, A_{i}$ is larger if the fluid- $i$ is wetting to the solid surface. As a consequence, if we define for each of the fluid phases $i$ the fluid- $i$-coated area fraction as:

$$
A_{f i}\left(S_{i}\right)=\frac{A_{i}\left(S_{i}\right)}{\sum_{i} A_{i}\left(S_{i}\right)}
$$

given a certain fluid saturation $S_{i}, A_{f i}\left(S_{i}\right)$ will be larger if the solid surface is wetting to $i$ or smaller if it is not. Garfi et al. $(2020)^{25}$ have shown that the relative surface coverage is a signal of the wetting state and used this concept to identify mineral specific wetting behaviours in a sandstone rock sample.

\section{Generation of 3D wetting maps for intermediate and mixed-wet rock samples}

Figure 5 provides an illustratioun of how the wetting indices are quantitatively assigned to each pore using the data in Figure 2. The Bentheimer sandstone and Estaillades limestone are mineralogically homogeneous, comprising $>98 \mathrm{wt} . \%$ quartz or calcite, respectively. The unaltered samples are thus homogeneously hydrophilic and the curves in Figure 2a,c represent reference lower bounds in the graph for the hydrophilic state. Because the pore structure does not change, we can rotate this curve around the $1: 1$ line to similarly create an upper bounding curve representative of a reference hydrophobic state in the rocks. The thermodynamic basis for this is described in the previous section and an example analytical model created using Brooks-Corey curves is provided in Eq.10 and 11.

To avoid requiring a specific analytical form of the capillary pressure characteristic curve, we create an empirical model for the bounding curves based on the data from the unaltered rock samples. We use twice the standard deviation from the mean as a lower bounding value of the oil coated area fraction. We fit a third order polynomial to those values.

For each bin $k$,

$$
A_{f o, L B}^{k}=\mu_{A_{f o}}^{k}-2 \sigma_{A_{f o}}^{k}
$$




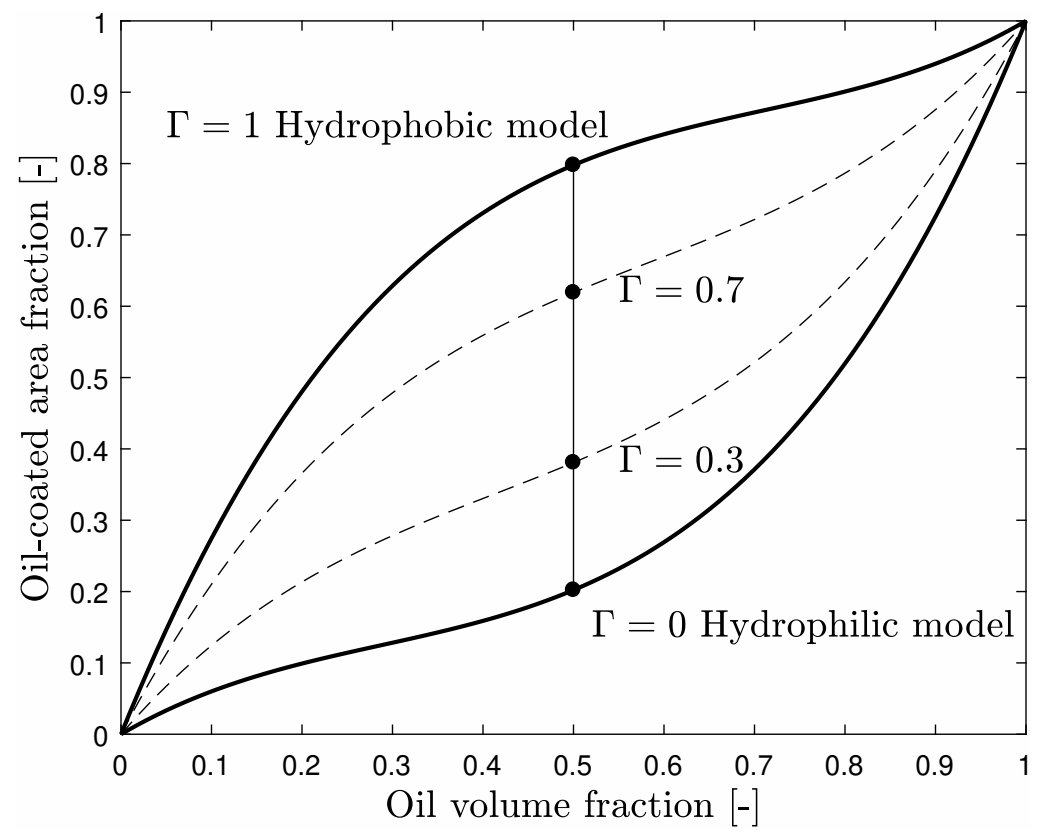

Figure 5. Conceptual approach to the definition of the wetting index $\Gamma$ for the altered Bentheimer. The approach is analogous for the altered Estaillades. The empirical model defining the hydrophilic reference case is defined by fitting a third order polynomial to the hydrophilic binned data. The hydrophobic reference model is defined from the hydrophilic one by performing a rigid translation and reflection with respect to the 1:1 line. $\Gamma$ ranges from 0 for the reference hydrophilic case to 1 for the reference hydrophobic case.

where $\mu_{A_{f o}}$ and $\sigma_{A_{f o}}^{k}$ are the mean and standard deviation of the oil area fraction in the bin. Values of $A_{f o, L B}^{k}$ are fit by a third order polynomial forced through $(0,0)$ and $(1,1)$, i.e. $p(x)=a x^{3}+b x^{2}+c x$. Following from the thermodynamic theory, the upper bounding wetting state model, i.e., the one defining a reference hydrophobic pore is defined by performing a reflection of $p(x)$ with respect to the $y$-axis and a rigid translation, obtaining $q(x)=-a(1-x)^{3}-b(1-x)^{2}-c(1-x)+1$. This procedure was employed for obtaining the hydrophilic and hydrophobic models for altered Bentheimer and altered Estaillades. The estimated parameters for the polynomials for the Bentheimer are $a=1.87, b=-1.61$ and $c=0.74$.

Once the reference bounding models are defined, for each pore- $i$ with oil volume fraction $0<V_{f o, i}<1$, the wetting index $\Gamma_{i}$ is defined by

$$
\Gamma_{i}=\frac{A_{f o, i}-p\left(V_{f o, i}\right)}{q\left(V_{f o, i}\right)-p\left(V_{f o, i}\right)}
$$

where $p\left(V_{f o, i}\right)$ and $q\left(V_{f o, i}\right)$ are the bounding experimental polynomials evaluated in $V_{f o, i}$. If $A_{f o, i} \geq q\left(V_{f o, i}\right), \Gamma_{i} \triangleq 1$; if $A_{f o, i} \leq p\left(V_{f o, i}\right) ., \Gamma_{i} \triangleq 0$. The wetting index $\Gamma$ thus ranges from 0 for pores equally or more hydrophilic than the reference case to 1 for pores equally or more hydrophobic than the reference hydrophobic case.

\section{References}

1. Cavanagh, A. J. \& Haszeldine, R. S. The sleipner storage site: Capillary flow modeling of a layered co2 plume requires fractured shale barriers within the utsira formation. Int. J. Greenh. Gas Control. 21, 101-112, DOI: https: //doi.org/10.1016/j.ijggc.2013.11.017 (2014).

2. McCray, J. E. \& Falta, R. W. Numerical simulation of air sparging for remediation of napl contamination. Groundwater 35, 99-110, DOI: https://doi.org/10.1111/j.1745-6584.1997.tb00065.x (1997). https://ngwa.onlinelibrary.wiley.com/doi/ pdf/10.1111/j.1745-6584.1997.tb00065.x.

3. DiCarlo, D. A. Experimental measurements of saturation overshoot on infiltration. Water Resour. Res. 40, DOI: https://doi.org/10.1029/2003WR002670 (2004). https://agupubs.onlinelibrary.wiley.com/doi/pdf/10.1029/2003WR002670.

4. Christie, M. A. \& Blunt, M. J. Tenth SPE Comparative Solution Project: A Comparison of Upscaling Techniques, DOI: $10.2118 / 66599-\mathrm{MS}$ (2001). 
5. Zhao, B. et al. Comprehensive comparison of pore-scale models for multiphase flow in porous media. Proc. Natl. Acad. Sci. 116, 13799-13806, DOI: 10.1073/pnas.1901619116 (2019). https://www.pnas.org/content/116/28/13799.full.pdf.

6. Blunt, M. J. Multiphase Flow in Permeable Media. DOI: 10.1017/9781316145098 (2017).

7. Bear, J. Dynamics of fluids in porous media. Soil Sci. 120, 162-163 (1972).

8. Bultreys, T., De Boever, W. \& Cnudde, V. Imaging and image-based fluid transport modeling at the pore scale in geological materials: A practical introduction to the current state-of-the-art. Earth-Science Rev. 155, 93-128, DOI: 10.1016/j.earscirev.2016.02.001 (2016). 1209.3974.

9. Raeini, A. Q., Blunt, M. J. \& Bijeljic, B. Direct simulations of two-phase flow on micro-ct images of porous media and upscaling of pore-scale forces. Adv. Water Resour. 74, 116-126, DOI: https://doi.org/10.1016/j.advwatres.2014.08.012 (2014).

10. Blunt, M. J. et al. Pore-scale imaging and modelling. Adv. Water Resour. 51, 197-216, DOI: 10.1016/j.advwatres.2012.03. 003 (2013)

11. Yang, J. \& Boek, E. S. A comparison study of multi-component lattice boltzmann models for flow in porous media applications. Comput. Math. with Appl. 65, 882-890, DOI: https://doi.org/10.1016/j.camwa.2012.11.022 (2013). Mesoscopic Methods in Engineering and Science.

12. Miller, C. T., Bruning, K., Talbot, C. L., McClure, J. E. \& Gray, W. G. Nonhysteretic capillary pressure in two-fluid porous medium systems: Definition, evaluation, validation, and dynamics. Water Resour. Res. 55, 6825-6849, DOI: https://doi.org/10.1029/2018WR024586 (2019). https://agupubs.onlinelibrary.wiley.com/doi/pdf/10.1029/2018WR024586.

13. McClure, J. E. et al. Geometric state function for two-fluid flow in porous media. Phys. Rev. Fluids 3, 084306, DOI: 10.1103/PhysRevFluids.3.084306 (2018).

14. Bultreys, T. et al. Validation of model predictions of pore-scale fluid distributions during two-phase flow. Phys. Rev. E 97, DOI: 10.1103/PhysRevE.97.053104 (2018).

15. Liu, Z., Herring, A., Arns, C., Berg, S. \& Armstrong, R. T. Pore-Scale Characterization of Two-Phase Flow Using Integral Geometry. Transp. Porous Media 118, 99-117, DOI: 10.1007/s11242-017-0849-5 (2017).

16. Armstrong, R. T. et al. Beyond darcy's law: The role of phase topology and ganglion dynamics for two-fluid flow. Phys. Rev. E 94, 043113, DOI: 10.1103/PhysRevE.94.043113 (2016).

17. Rücker, M. et al. From connected pathway flow to ganglion dynamics. Geophys. Res. Lett. 42, 3888-3894, DOI: https://doi.org/10.1002/2015GL064007 (2015). https://agupubs.onlinelibrary.wiley.com/doi/pdf/10.1002/2015GL064007.

18. Murison, J. et al. Wetting Heterogeneities in Porous Media Control Flow Dissipation. Phys. Rev. Appl. 2, 1-10, DOI: 10.1103/PhysRevApplied.2.034002 (2014). arXiv:1310.3125v2.

19. Andrew, M., Bijeljic, B. \& Blunt, M. J. Pore-scale contact angle measurements at reservoir conditions using X-ray microtomography. Adv. Water Resour. 68, 24-31, DOI: 10.1016/j.advwatres.2014.02.014 (2014).

20. Klise, K. A., Moriarty, D., Yoon, H. \& Karpyn, Z. Automated contact angle estimation for three-dimensional X-ray microtomography data. Adv. Water Resour. 95, DOI: 10.1016/j.advwatres.2015.11.006 (2016).

21. Scanziani, A., Singh, K., Blunt, M. J. \& Guadagnini, A. Automatic method for estimation of in situ effective contact angle from X-ray micro tomography images of two-phase flow in porous media. J. Colloid Interface Sci. 496, 51-59, DOI: 10.1016/j.jcis.2017.02.005 (2017).

22. AlRatrout, A., Raeini, A. Q., Bijeljic, B. \& Blunt, M. J. Automatic measurement of contact angle in pore-space images. Adv. Water Resour. 109, 158-169, DOI: 10.1016/j.advwatres.2017.07.018 (2017).

23. Garfi, G., John, C. M., Berg, S. \& Krevor, S. The Sensitivity of Estimates of Multiphase Fluid and Solid Properties of Porous Rocks to Image Processing. Transp. Porous Media DOI: 10.1007/s11242-019-01374-z (2019).

24. Blunt, M. J., Lin, Q., Akai, T. \& Bijeljic, B. A thermodynamically consistent characterization of wettability in porous media using high-resolution imaging. J. Colloid Interface Sci. 552, 59-65, DOI: https://doi.org/10.1016/j.jcis.2019.05.026 (2019).

25. Garfi, G., John, C. M., Lin, Q., Berg, S. \& Krevor, S. Fluid surface coverage showing the controls of rock mineralogy on the wetting state. Geophys. Res. Lett. 47, e2019GL086380, DOI: 10.1029/2019GL086380 (2020). E2019GL086380 10.1029/2019GL086380, https://agupubs.onlinelibrary.wiley.com/doi/pdf/10.1029/2019GL086380.

26. Sun, C. et al. Characterization of wetting using topological principles. J. Colloid Interface Sci. 578, 106 - 115, DOI: https://doi.org/10.1016/j.jcis.2020.05.076 (2020). 
27. Foroughi, S., Bijeljic, B., Lin, Q., Raeini, A. Q. \& Blunt, M. J. Pore-by-pore modeling, analysis, and prediction of two-phase flow in mixed-wet rocks. Phys. Rev. E 102, 23302, DOI: 10.1103/PhysRevE.102.023302 (2020).

28. Valvatne, P. H. \& Blunt, M. J. Predictive pore-scale modeling of two-phase flow in mixed wet media. Water Resour. Res. 40, DOI: https://doi.org/10.1029/2003WR002627 (2004). https://agupubs.onlinelibrary.wiley.com/doi/pdf/10.1029/ 2003WR002627.

29. Mularczyk, A. et al. Droplet and Percolation Network Interactions in a Fuel Cell Gas Diffusion Layer. J. The Electrochem. Soc. 167, 084506, DOI: 10.1149/1945-7111/ab8c85 (2020).

30. Shrestha, P. et al. Graded microporous layers for enhanced capillary-driven liquid water removal in polymer electrolyte membrane fuel cells. Adv. Mater. Interfaces 6, 1901157, DOI: https://doi.org/10.1002/admi.201901157 (2019). https: //onlinelibrary.wiley.com/doi/pdf/10.1002/admi.201901157.

31. Akai, T., Alhammadi, A., Blunt, M. \& Bijeljic, B. Modeling oil recovery in mixed-wet rocks: Pore-scale comparison between experiment and simulation. Transp. Porous Media 127, DOI: 10.1007/s11242-018-1198-8 (2019).

32. Lin, Q., Bijeljic, B., Pini, R., Blunt, M. J. \& Krevor, S. Imaging and Measurement of Pore-Scale Interfacial Curvature to Determine Capillary Pressure Simultaneously With Relative Permeability. 7046-7060, DOI: 10.1029/2018WR023214 (2018).

33. Lin, Q. et al. Minimal surfaces in porous media: Pore-scale imaging of multiphase flow in an altered-wettability bentheimer sandstone. Phys. Rev. E 99, 063105, DOI: 10.1103/PhysRevE.99.063105 (2019).

34. Alhammadi, A. M., AlRatrout, A., Singh, K., Bijeljic, B. \& Blunt, M. J. In situ characterization of mixed-wettability in a reservoir rock at subsurface conditions. Sci. Reports 7, 10753, DOI: 10.1038/s41598-017-10992-w (2017).

35. Alipour Tabrizy, V., Denoyel, R. \& Hamouda, A. A. Characterization of wettability alteration of calcite, quartz and kaolinite: Surface energy analysis. Colloids Surfaces A: Physicochem. Eng. Aspects 384, 98-108, DOI: 10.1016/j.colsurfa. 2011.03.021 (2011).

36. Lin, Q. et al. A New Waterflood Initialization Protocol With Wettability Alteration for Pore-Scale Multiphase Flow Experiments. Petrophysics 60, 264-272, DOI: 10.30632/PJV60N2-2019a4 (2019).

37. Rücker, M. et al. Relationship between wetting and capillary pressure in a crude oil/brine/rock system: From nano-scale to core-scale. J. Colloid Interface Sci. 562, 159-169, DOI: https://doi.org/10.1016/j.jcis.2019.11.086 (2020).

38. Lin, Q., Bijeljic, B., Foroughi, S., Berg, S. \& Blunt, M. J. Pore-scale imaging of displacement patterns in an alteredwettability carbonate. Chem. Eng. Sci. 235, 116464, DOI: https://doi.org/10.1016/j.ces.2021.116464 (2021).

39. Bultreys, T. et al. Investigating the relative permeability behavior of microporosity-rich carbonates and tight sandstones with multiscale pore network models. J. Geophys. Res. Solid Earth 121, 7929-7945, DOI: https://doi.org/10.1002/2016JB013328 (2016). https://agupubs.onlinelibrary.wiley.com/doi/pdf/10.1002/2016JB013328.

40. Morrow, N. R. Wettability and Its Effect on Oil Recovery. J. Petroleum Technol. 42, 1476-1484, DOI: 10.2118/21621-PA (1990).

41. Buckley, J. S. \& Liu, Y. Some mechanisms of crude oil/brine/solid interactions. Petroleum Sci. Eng. 155-160 (1998).

42. Buckley, J. S. Wetting Alteration of Solid Surfaces by Crude Oils and Their Asphaltenes. Revue de l'Institut Français du Pétrole 53, 303-312, DOI: 10.2516/ogst:1998026 (1998).

43. Beucher, S. \& Meyer, F. The morphological approach to segmentation: the watershed transformation. Math. Morphol. Image Process. 433-481, DOI: ExportDate6May2013 (1993).

44. Lin, Q., Al-khulaifi, Y., Blunt, M. J. \& Bijeljic, B. Advances in Water Resources Quantification of sub-resolution porosity in carbonate rocks by applying high-salinity contrast brine using X-ray microtomography differential imaging. Adv. Water Resour. 96, 306-322, DOI: 10.1016/j.advwatres.2016.08.002 (2016).

45. Lin, Q., Bijeljic, B., Berg, S. \& Blunt, M. Data for pore-scale imaging of displacement patterns in an altered-wettability carbonate. http://www.digitalrocksportal.org/projects/331, DOI: 10.17612/XWVW-9E52 (2020).

46. Wildenschild, D. \& Sheppard, A. P. X-ray imaging and analysis techniques for quantifying pore-scale structure and processes in subsurface porous medium systems. Adv. Water Resour. 51, 217-246, DOI: 10.1016/j.advwatres.2012.07.018 (2013).

47. Bultreys, T. et al. Fast laboratory-based micro-computed tomography for pore-scale research: Illustrative experiments and perspectives on the future. Adv. Water Resour. 95, 341-351, DOI: 10.1016/j.advwatres.2015.05.012 (2016). 
48. Dong, H. \& Blunt, M. J. Pore-network extraction from micro-computerized-tomography images. Phys. Rev. E 80, 036307, DOI: 10.1103/PhysRevE.80.036307 (2009).

49. Raeini, A. Q., Bijeljic, B. \& Blunt, M. J. Generalized network modeling : Network extraction as a coarse-scale discretization of the void space of porous media. 013312, 1-17, DOI: 10.1103/PhysRevE.96.013312 (2017).

50. Taubin, G. Curve and surface smoothing without shrinkage. Proc. IEEE Int. Conf. on Comput. Vis. $852-857$ (1995).

51. Bradford, S. A. \& Leij, F. J. Estimating interfacial areas for multi-fluid soil systems. J. Contaminant Hydrol. 27, 83-105, DOI: https://doi.org/10.1016/S0169-7722(96)00048-4 (1997).

52. Morrow, N. R. \& Szabo, J. O. Physics and Thermodynamics of Capillary. Ind. Eng. Chem. 62, 32-56, DOI: 10.1021/ ie50726a006 (1970).

53. Porter, M. L., Schaap, M. G. \& Wildenschild, D. Lattice-Boltzmann simulations of the capillary pressure-saturation-interfacial area relationship for porous media. Adv. Water Resour. 32, 1632-1640, DOI: 10.1016/j. advwatres.2009.08.009 (2009).

54. Joekar-Niasar, V. \& Hassanizadeh, S. M. Uniqueness of Specific Interfacial Area-Capillary Pressure-Saturation Relationship Under Non-Equilibrium Conditions in Two-Phase Porous Media Flow. Transp. Porous Media 94, 465-486, DOI: 10.1007/s11242-012-9958-3 (2012).

\section{Acknowledgements}

We gratefully acknowledge Shell Global Solutions International B.V. for funding this work. We thank Edward Andrews and Conxita Taberner for the useful discussions.

\section{Author contributions statement}

G.G. and S.K. conceived the study, developed the thermodynamic theory, and analysed the data. G.G., S.K., M.R. and Q.L. conceived the unaltered Estaillades experiment. G.G., C.S. and Q.L. conducted the experiment. G.G., S.K., C.M.J. and S.B. conceived the first draft of the manuscript. G.G. and S.K. wrote the paper. All authors equally contributed to review the manuscript.

\section{Additional information}

Competing interests: the authors declare no competing interests. 